\title{
Application of Fuzzy Synthetic Evaluation Method in Poor University Student Entrepreneurship Evaluation
}

\author{
Yongmei $\mathrm{Hao}^{1}$, Meihong Chen ${ }^{1}$ \\ ${ }^{1}$ Nanjing University of Information Science and Technology, Nanjing, 210044, China
}

Keywords: Poor students, Entrepreneurship, Supporting system, Evaluation

\begin{abstract}
The relative weakness of poor university students in employment attracts more and more attention, and how to encourage poor students to become self-employed and ease the employment tension is a problem highly concerned by the whole society. This paper applies the fuzzy synthetic evaluation method in the poor university student entrepreneurship evaluation, to establish the poor university student entrepreneurship evaluation index system and model. Meanwhile, this paper puts forward opinions and suggestions by carrying out the case analysis on poor student entrepreneurship evaluation index system of a university.
\end{abstract}

\section{Introduction}

In recent years, the university student entrepreneurship has attracted the high attention from all sectors of the society, and governments at all levels and universities strong support university students' self-employment, and have released many preferential policies to promote university student entrepreneurship; for example, in 2008, the Ministry of Human Resources and Social Security, the Ministry of Education and other ministries and commissions pointed out in the Guiding Opinions for Promoting Employment by Entrepreneurship that "main guidance should be provided for university graduates to strive for achieving, within 3-5 years, the substantial increase in the quantity of worker entrepreneurship and the number of employment by self-employment, to basically form the policy system that drives the employment by entrepreneurship, and to make workers with entrepreneurial intention and ability succeeded in entrepreneurship". Poor university students are an inevitable group among university students and are even a special team among university students entering the workplace; their employment pressure is getting greater due to relevant factors such as severe challenge by employment situation, constraint by economic poverty, influence by mental poverty, lack of social resources, restraint by comprehensive quality, etc. Encouraging, supporting and helping poor university students to start their businesses is an important content of positively implementing the preferential policies for entrepreneurship, and is not only a long-term mechanism for employment improvement, but also beneficial to expanding employment channels of poor university students.

A series of problems emerge in front of poor university students, such as how to start a business, how to draw the support by the society, governments and universities, how to start their businesses in the condition of Chinese market economy, etc.; therefore, the establishment and improvement of poor university student entrepreneurship supporting system become particularly important; the poor university student entrepreneurship supporting system plays a key role in poor student entrepreneurship, and many scholars have made a lot of corresponding researches on poor university student entrepreneurship and university student entrepreneurship supporting system. Chen Long (2010) proposed the establishment of the university student entrepreneurship supporting system that consists of four subsystems, namely the government support, educational support, fund support and incubation support, so as to support and serve university students' entrepreneurship and perform the fuzzy evaluation on the university student entrepreneurship supporting system; he also gave corresponding opinions and suggestions ${ }^{[1]}$. Xiao Li (2010) selected a poor university student as an individual case to analyze the main difficulties faced by poor graduates during entrepreneurship, and to explore, on this basis, how poor university students solve such entrepreneurship difficulties ${ }^{[2]}$. Guo Ling (2013) started from the investigation data about poor students' entrepreneurship qualities to 
analyze the entrepreneurial intention, knowledge, quality and current environment of poor students, provided corresponding suggestions and countermeasures ${ }^{[3]}$. In recent years, some scholars have also provided their suggestions and evaluation on the establishment of domestic university student entrepreneurship supporting system by comparative analysis on foreign entrepreneurship groups; however, due to the particularity of poor students, there are few evaluations on the poor university student entrepreneurship supporting system. The writer tries to perform the fuzzy synthetic evaluation on the poor university student entrepreneurship supporting system on the basis of scholars' researches and with the help of the fuzzy hierarchical analysis method, and puts forward corresponding suggestions and conclusions.

\section{Fuzzy synthetic evaluation model ${ }^{[4]}$}

The fuzzy synthetic evaluation method is a systematic analysis method that applies the fuzzy mathematics principle to analyze and evaluate "fuzzy" things, and is a analytical evaluation method that relies mainly on fuzzy inference, integrates quality and quantity, and combines precision and imprecision. As this method shows its unique superiority in dealing with complicated systematic problems that cannot be described with precise mathematical methods, it has been widely applied in many disciplines in recent years.

Evaluation model and evaluation procedure:

Assuming that the evaluation object is $A$ : its factors set $B=\left\{b_{1}, b_{2}, \ldots, b_{n}\right\}$, evaluation grades set $C=\left\{c_{1}, c_{2}, \ldots, c_{m}\right\}$; the fuzzy evaluation is performed on each factor in $B$ according to the grade index in the evaluation grades set, to obtain the evaluation matrix:

$$
D=\left[\begin{array}{llll}
d_{11} & d_{12} & \ldots & d_{1 m} \\
d_{21} & d_{22} & \ldots & d_{2 m} \\
\ldots & & & \\
d_{n 1} & d_{n 2} & \ldots & d_{n m}
\end{array}\right]
$$

Where: $d_{i j}$ means the subjection degree of $b_{i}$ related to $c_{j}$. Thus, $(B, C, D)$ constitutes a fuzzy synthetic evaluation model. After determining the importance index (also called weight) of each factor, the $E=\left\{e_{1}, e_{2}, \ldots, e_{n}\right\}$ is obtained, satisfying $\sum_{i=1}^{n} e_{i}=1$; after the synthesis, the $F=E \cdot D=\left\{f_{1}, f_{2}, \ldots, f_{m}\right\}$ is obtained; then, the evaluation grade of object $A$ can be determined.

\section{Case analysis}

With the fuzzy synthetic evaluation method, the writer takes a university in Jiangsu as an example to perform the fuzzy synthetic evaluation on poor university student entrepreneurship supporting system. The selection of all levels of indicators for poor university student entrepreneurship evaluation system needs to follow the principles of objectivity, comprehensiveness and operability; by combining the actual situation of the selected university and previous researches by scholars, the poor university student entrepreneurship evaluation system can be established. This system consists of 4 level-I indexes, namely the policy support, educational support, fund support and incubation support, which include 16 level-II indexes. (see Table 1) 
Table 1. Poor University Student Entrepreneurship Evaluation Index System A

\begin{tabular}{|c|c|c|c|c|c|}
\hline \multirow{2}{*}{$\begin{array}{c}\text { Level-I Index and } \\
\text { Weight }\end{array}$} & \multirow{2}{*}{ Level-II Index and Weight } & \multicolumn{4}{|c|}{ Evaluation Grade and Weight } \\
\hline & & Excellent & Good & Ordinary & Poor \\
\hline \multirow{4}{*}{$\begin{array}{c}\text { Policy support } B_{1} \\
0.30\end{array}$} & $\begin{array}{l}\text { Entrepreneurship } \\
\text { encouragement } B_{11} 0.13\end{array}$ & 0.42 & 0.31 & 0.27 & 0 \\
\hline & $\begin{array}{l}\text { Tax reduction or exemption } \\
\qquad B_{12} \quad 0.35 \\
\end{array}$ & 0.19 & 0.25 & 0.45 & 0.11 \\
\hline & Technical support $B_{13} \quad 0.32$ & 0.07 & 0.13 & 0.62 & 0.18 \\
\hline & Market service $B_{14} \quad 0.20$ & 0.59 & 0.27 & 0.11 & 0.03 \\
\hline \multirow{4}{*}{$\begin{array}{c}\text { Educational support } \\
\qquad B_{2} \\
0.30\end{array}$} & Organization $B_{21} \quad 0.16$ & 0.05 & 0.12 & 0.15 & 0.68 \\
\hline & Teaching system $B_{22} \quad 0.35$ & 0.03 & 0.12 & 0.37 & 0.48 \\
\hline & $\begin{array}{c}\text { Operation mechanism } B_{23} \\
0.28 \\
\end{array}$ & 0.02 & 0.04 & 0.10 & 0.84 \\
\hline & $\begin{array}{l}\text { Entrepreneurship competition } \\
\qquad \begin{array}{cc}B_{24} & 0.21 \\
\end{array}\end{array}$ & 0.10 & 0.15 & 0.52 & 0.23 \\
\hline \multirow{4}{*}{$\begin{array}{c}\text { Fund support }{ }^{B_{3}} \\
0.20\end{array}$} & Family support $B_{31} \quad 0.05$ & 0.01 & 0.03 & 0.11 & 0.85 \\
\hline & Bank support $B_{32} \quad 0.56$ & 0.21 & 0.23 & 0.44 & 0.12 \\
\hline & School support $B_{33} \quad 0.12$ & 0.12 & 0.17 & 0.42 & 0.29 \\
\hline & Social support $B_{34} \quad 0.27$ & 0.14 & 0.15 & 0.45 & 0.26 \\
\hline \multirow{4}{*}{$\begin{array}{c}\text { Incubation support } \\
B_{4} \\
0.20\end{array}$} & Entrepreneurial base $B_{41} \quad 0.35$ & 0.04 & 0.10 & 0.16 & 0.70 \\
\hline & $\begin{array}{l}\text { Entrepreneurial equipment } \\
\qquad B_{42} \quad 0.25 \\
\end{array}$ & 0.04 & 0.08 & 0.12 & 0.76 \\
\hline & Channel guidance $B_{43} \quad 0.20$ & 0.10 & 0.12 & 0.23 & 0.55 \\
\hline & Management service $B_{44} \quad 0.20$ & 0.08 & 0.09 & 0.15 & 0.68 \\
\hline
\end{tabular}

\section{Design the weights of level-I and level-II indexes}

Considering the importance of all levels of evaluation indexes, according to the previous analyses by scholars and the hierarchical analysis method, each level-I index and level-II index are given with the weight proportion. The weight of each index is as follows:

Weight of level-I index is: $A=(0.30,0.30,0.20,0.20)$

Weights of level-II indexes are respectively:

$$
\begin{aligned}
& B_{2}=(0.16,0.35,0.28,0.21) \\
& B_{3}=(0.05,0.56,0.12,0.27) \\
& B_{4}=(0.35,0.25,0.20,0.20)
\end{aligned}
$$

Meanwhile, the Excellent, Good, Ordinary, and Poor in Table 1 are corresponding to the scores 95, 85, 70, and 40 respectively; thus, it can be learned that the 4 grades of quantized indexes are $C=\left(c_{1}, c_{2}, c_{3}, c_{4}\right)=(95,85,70,40)$.

\section{Calculate the fuzzy synthetic evaluation matrix}

Vectorize the evaluation model to obtain specific numerical value for sequencing and comparison.

(1) Calculate the subjection vector $E_{i}$ of level-I index layer $B_{i}$ corresponding to grade $C$; 
Thus:

$$
E_{i}=B_{i} \times D_{i}=\left(E_{i 1}, E_{i 2}, \ldots, E_{i m}\right)
$$

$$
\begin{aligned}
& E_{1}=B_{1} \times D_{1}=(0.13,0.35,0.32,0.20) \times\left[\begin{array}{cccc}
0.42 & 0.31 & 0.27 & 0 \\
0.19 & 0.25 & 0.45 & 0.11 \\
0.07 & 0.13 & 0.62 & 0.18 \\
0.59 & 0.27 & 0.11 & 0.13
\end{array}\right] \\
& =(0.2615,0.2234,0.413,0.1021)
\end{aligned}
$$

The result indicates that the $26.15 \%$ of policy support for poor university student entrepreneurship evaluation index system has a very remarkable effect, the $22.34 \%$ has a remarkable effect, the $41.3 \%$ has a common effect, and the 10.21 has an indistinctive effect.

Similarly,

$$
\begin{gathered}
E_{2}=B_{2} \times D_{2}=(0.0451,0.1039,0.2907,0.5603) \\
E_{3}=B_{3} \times D_{3}=(0.1703,0.1912,0.4238,0.2147) \\
E_{4}=B_{4} \times D_{4}=(0.06,0.097,0.162,0.681)
\end{gathered}
$$

(2) Perform the score evaluation on the index $B_{i j}$ in the level-II index layer; then, the effect of policy support is divided into:

$$
\begin{aligned}
F_{1}= & E_{1} \times C^{T}=(0.2615,0.2234,0.413,0.1021) \times(95,85,70,40)^{T} \\
& =76.8255
\end{aligned}
$$

Similarly, $F_{2}=E_{2} \times C^{T}=55.877$

$$
\begin{aligned}
& F_{3}=E_{3} \times C^{T}=70.6845 \\
& F_{4}=E_{4} \times C^{T}=52.525
\end{aligned}
$$

\section{Build the fuzzy synthetic evaluation matrix}

The fuzzy synthetic evaluation matrix $D$ is used to express the relations among level-I and level-II indexes and effect, where the factor $D_{i j}$ in $D$ means the subjection degree of index $B_{i}$ related to $C_{j}$.

According to the matrix operation method,

$$
D=\left(E_{1}, E_{2}, E_{3}, E_{4}\right)^{T}=\left[\begin{array}{cccc}
0.2615 & 0.2234 & 0.413 & 0.1021 \\
0.0451 & 0.1039 & 0.2907 & 0.5603 \\
0.1703 & 0.1912 & 0.4238 & 0.2147 \\
0.06 & 0.097 & 0.162 & 0.681
\end{array}\right]
$$

$$
\begin{aligned}
E=A \times D & =(0.30,0.30,0.20,0.20) \times\left(E_{1}, E_{2}, E_{3}, E_{4}\right)^{T} \\
& =(0.13804,0.15583,0.32827,0.37786)
\end{aligned}
$$

\section{Calculate the scores of evaluation results}

The quantization effect of index system evaluation can be obtained by the matrix calculated by quantizing the given index weight multiplying the matrix formed with the evaluated value of each index in grade $C$, namely:

$$
F=E \times C^{T}=(0.13804,0.15583,0.32827,0.37786) \times(95,85,70,40)^{T}=64.45
$$

Namely, the effect score of a poor university student entrepreneurship evaluation index system is 64.45 . 


\section{Conclusions and suggestions}

\section{The support for poor university student entrepreneurship is not fully implemented}

It can be learned from the synthetic evaluation model $E_{1}$ that the proportion of common effect is $41.3 \%$, much higher than that of very remarkable effect and that of remarkable effect, which indicates that the each sub-index layer in the poor university student entrepreneurship supporting system has actually functioned very well, but there are still some problems between sub-index layers, such as non-coordination and insufficient implementation. Currently, the state and all levels of governments have issued a series of policies to encourage poor students to start their businesses, but departments fail to implement such policies consistently and to have a smooth communication; the formalities are also complex. Governments at all levels must transform their functions, further improve various supporting facilities, establish training, service, financing and educational platforms, coordinate the taxation, industry and commerce, finance and other relevant parts, sufficiently implement preferential policies for university student entrepreneurship, open the "green channels" and simplify the formalities, to provide rich external environment support for poor university student entrepreneurship.

The educational support for poor university student entrepreneurship needs to be improved

It can be learned from the $E_{2}$ single-layer evaluation that the proportion of indistinctive effect is $56.03 \%$, much higher than that of very remarkable effect and that of remarkable effect, which indicates that the educational support plays a indistinctive role in the poor university student entrepreneurship. Compared with European and American countries with mature development of university student entrepreneurship education, our country provides restricted conditions for university student entrepreneurship, especially for poor university student entrepreneurship; due to the lack of teachers for entrepreneurship education, insufficient assisting funds for entrepreneurship education, imperfect entrepreneurship education course system and incomplete relevant mechanism in entrepreneurship education institutions, the systematic support and long-term mechanism have not been formed yet. To serve the poor university student entrepreneurship in a better way, universities need to establish a rational entrepreneurship education course system, set up professional entrepreneurship education institutions, create the entrepreneurship platform replying on the entrepreneurial base, and construct the "entrepreneurship mentor" team. The success of poor university student entrepreneurship is not only restricted by students themselves, and the positive guidance and facilitation by universities will bring an effect of doing less for more.

\section{The fund for poor university student entrepreneurship is insufficient}

It can be learned from the $E_{3}$ single-layer evaluation that the proportion of common effect is 42.38\%, higher than that of very remarkable effect and that of remarkable effect, which indicates that the insufficient fund is still an inevitable obstacle for poor university student entrepreneurship. Although the state and local governments at all levels have issued preferential policies for university students to obtain entrepreneurship loans, many poor students perish the thought of entrepreneurship during the practical operation due to the loan limit or complex formalities. As a special group among university students, poor students face a very great employment pressure. As they are limited by the living and educational environment since their childhood, some small frustrations in the process when they initially have the though of entrepreneurship will often bring them with negative thoughts. In order to support the poor university student entrepreneurship in a better way, the governments need to strengthen the financial input, simplify the loan application procedure, set up the financing indexes aimed at poor student entrepreneurship, positively guide the social funds to support the poor student entrepreneurship, and expand source channels of funds for poor student entrepreneurship.

The effect of incubation support is indistinctive for poor university student entrepreneurship

It can be learned from the $E_{4}$ single-layer evaluation that the proportion of indistinctive effect is $68.1 \%$, much higher than that of very remarkable effect, that of remarkable effect and that of common effect, which indicates that the entrepreneurship practice is not implemented sufficiently. Currently, 
many universities' support for poor student entrepreneurship stays on the theoretical layer; they do not have a special site as the poor student entrepreneurship incubator and lack the basic office equipment, which bring software and hardware disadvantages for poor student entrepreneurship. In order to provide the incubation support for poor student entrepreneurship, universities and local places shall take the lead to create the entrepreneurial park, help poor students to deal with a series of matters such as registration in the early-stage entrepreneurship, financing, enterprise diagnosis, employee recruitment, etc., to ease their pressure and encourage their entrepreneurship. It is also needed to provide poor students with certain support in the aspects of market situation, client information, customer resources, etc., to assist them in completing the market operation and achieving the more efficient, convenient and ordered company operation.

\section{Conclusion}

The poor university student entrepreneurship supporting system is a social systematic project, and the entrepreneurship standardization and systematization can be promoted only by government leading, social support, university participation, family support, and establishing the diversified and three-dimensional entrepreneurship supporting system. The evaluation object of poor university student entrepreneurship is the entrepreneur or the entrepreneurial team, and the evaluation subjects universities and risk investors; it is a theoretical system that must be established when the poor university student entrepreneurship rises to a certain stage. Establishing a rational poor university student entrepreneurship education supporting system has become an urgent thing for poor students to have "the third educational passport". The writer believes that more poor students will join in the team of entrepreneurs along with the continuous improvement of the poor university student entrepreneurship supporting system in our country, which will not only ease the employment pressure, but also greatly promote the sustainable development of the society and economy.

\section{Acknowledgments}

This paper is a guiding project for philosophy and social science research in Colleges and Universities in Jiangsu; the project name is: Research on Innovation of university Career-creating Talent Cultivation Mode with Industry Characteristics, and the project number is: 2012SJDFDY053. It is also a key determined project for special research on entrepreneurship of Nanjing University of Information Science \& Technology in 2014; the project name is: Research on Poor University Student Identification Method and Subsidization System Based on Fuzzy Synthetic Evaluation, and the project number is: 2014NUISTZZB04.

\section{References}

[1] Chen Long, Zhu Yonghua and Liu Haibo. Evaluation on University Student Entrepreneurship Supporting System. Journal of Wuhan Institute of Technology, 2010 (6): 50-54.

[2] Xiao Li. Case Study on Difficulties for Poor University Student Entrepreneurship. Career Development, 2010 (2): 18-19.

[3] Guo Ling. Investigation on Quality Education for Poor University Student Entrepreneurship. Journal of Minjiang University, 2013 (1): 120-124.

[4] Cao Bingyuan. Application of Fuzzy Mathematics and System. Beijing: Science Press, 2005. 